TENDENCIAS

Revista de la Facultad de Ciencias

Económicas y Administrativas.

Universidad de Nariño

ISSN-E 2539-0554

Vol. XXII No. 2 - 2do Semestre 2021

Julio-Diciembre - Páginas 331-348

\title{
EL RETORNO DE LA INVERSIÓN EN MEDIOS SOCIALES (SOCIAL MEDIA ROI). UNA REVISIÓN DE LITERATURA
}

\section{RETURN ON INVESTMENT IN SOCIAL MEDIA. A LITERATURE REVIEW \\ O RETORNO DO INVESTIMENTO EM MÍDIAS SOCIAIS (SOCIAL MEDIA ROI). UMA REVISÃO DE LITERATURA}

\author{
Judith Vergara Garavito; Pilar Álvarez Franco; Maribel Serna Rodríguez
}

Doctora en Administración, CENTRUM Catolica Graduate Business School (CCGBS), Perú. Profesora Departamento de Finanzas, Escuela de Economía y Finanzas, Universidad EAFIT. ORCiD: 0000-0002-7466-944X. E-mail: jvergar8@eafit.edu.co, Colombia.

Doctora en Administración, CENTRUM Catolica Graduate Business School (CCGBS), Perú. Profesora Departamento de Finanzas, Escuela de Economía y Finanzas, Universidad EAFIT. ORCiD: 0000-0002-8557-6345. E-mail: palvare6@eafit.edu.co, Colombia.

Doctora en Administración, CENTRUM Catolica Graduate Business School (CCGBS), Perú. Profesora Departamento de Organización y Gerencia, Escuela de Administración, Universidad EAFIT. ORCiD: 0000- 0002-0349-9500. E-mail: mserna@eafit.edu.co, Colombia.

Recibido: 26 de junio de 2020

Aprobado: 29 de enero de 2021

DOI: https://doi.org/10.22267/rtend.212202.179

\section{Resumen}

En este trabajo se desarrolla una revisión de literatura del tema de retorno de la inversión en medios sociales (social media ROI). Fueron revisados artículos que en su mayoría corresponden a los Top Journals de marketing enfocándose, principalmente, en los últimos diez años en las bases de datos 
ISI Web of Science y Scopus. Como resultado de esta búsqueda, se logra dar cuenta del estado actual de lo que la literatura propone respecto a la estimación del ROI en medios sociales de comunicación, concluyendo que en ausencia del marketing tradicional y de las mejores prácticas estandarizadas, algunos recurren al retorno de la inversión (ROI) como medio para probar las contribuciones que los medios sociales generan, pese a que en el entorno de marketing parece no existir consenso respecto a lo que es el ROI.

Palabras clave: costos; desempeño financiero; marketing; rentabilidad; utilidades. JEL: D25; D92; E22; G11; G31

\begin{abstract}
This paper develops a literature review on the subject of return on investment in social media (social media ROI). Articles that mostly correspond to the Top Marketing Journals were reviewed, focusing mainly on the last ten years in the ISI Web of Science and Scopus databases. As a result of this search, it is possible to account for the current state of what the literature proposes regarding the estimation of ROI in social media, concluding that in the absence of traditional marketing and standardized best practices, some resort to the return of investment (ROI) as a means of testing the contributions that social media generates, despite the fact that in the marketing environment there seems to be no consensus on what ROI is.
\end{abstract}

Keywords: costs; financial performance; marketing; profitability; earnings.

JEL: D25; D92; E22; G11; G31

\title{
Resumo
}

Este artigo desenvolve uma revisão da literatura sobre o tema retorno do investimento em mídias sociais (ROI em mídias sociais). Os artigos que correspondem em sua maioria aos Top Marketing Journals foram revisados, com foco principalmente nos últimos dez anos nas bases de dados ISI Web of Science e Scopus. Como resultado desta pesquisa, é possível dar conta do estado atual do que a literatura propõe a respeito da estimativa do ROI nas mídias sociais, concluindo que na ausência de marketing tradicional e melhores práticas padronizadas, alguns recorrem ao retorno 
do investimento (ROI) como forma de testar as contribuições que a mídia social gera, apesar de no ambiente de marketing parecer não haver consenso sobre o que é ROI.

Palavras-chave: custos; desempenho financeiro; marketing; lucratividade; lucros.

JEL: D25; D92; E22; G11; G31

\section{Introducción}

En la última década tanto académicos como profesionales han sido testigos de una transformación importante del marketing, donde el rápido crecimiento de la adopción móvil ha abierto nuevas comunicaciones y posibilidades de focalización. Así mismo, la omnipresencia de las redes sociales ha cambiado la manera en que los compradores comparten información entre sí e interactúan con las marcas (Lamberton y Stephen, 2016). A medida que las marcas consolidan su lugar en los entornos de medios sociales, las expectativas de los consumidores se han amplificado, estimulando así el desarrollo de tecnologías para ayudar con el proceso de participación (Labrecque, 2014). El surgimiento de los nuevos medios está ayudando a los responsables del marketing a evolucionar desde el marketing digital al interactivo, lo que facilita una comunicación bidireccional entre los vendedores y los clientes sin inmiscuirse en su privacidad (Kumar et al., 2014).

A nivel organizacional, las plataformas de medios sociales como Facebook, Twitter, Digg, SCVNGR, YouTube y muchos otros, han comenzado a revolucionar el estado del marketing, la publicidad y las promociones, convirtiéndose no solo en plataformas de información, sino también en plataformas de influencia (Hanna et al., 2011). Además, la literatura reconoce que las redes sociales permiten a cualquier consumidor actuar como anunciante y consumidor de una marca determinada en cualquier momento (Lamberton y Stephen, 2016). De igual manera, el uso de los medios sociales a través del canal de distribución, ha generado un efecto contagio entre proveedores, minoristas y consumidores, lo que contribuye positivamente al desempeño de la marca y a la lealtad de los clientes (Rapp et al., 2013).

Si bien existen diferentes medios sociales de comunicación y su funcionalidad varía entre una aplicación y otra, éstos resultan atractivos para las empresas en su capacidad para permitir, 
fomentar y mejorar la participación, la conversación, la colaboración y la interacción (Weinberg y Pehlivan, 2011). La publicidad directa, entendida ésta como el envío de mensajes promocionales a clientes individuales, es también un mecanismo cada vez más utilizado por los vendedores como resultado de las recientes mejoras en la accesibilidad de los consumidores (Zubcsek y Sarvary, 2011). Dada su relevancia en el contexto del marketing y de las decisiones de inversión de una firma, en este trabajo se pretende dar cuenta del estado actual de lo que la literatura propone respecto a la estimación del ROI en medios sociales.

\section{Referentes teóricos}

La revisión de la literatura presentada en este trabajo se realizó considerando las diferentes contribuciones acerca de cómo se debe medir el ROI en medios sociales. Se presentan puntos de vista alternativos y diferentes enfoques. La literatura compara y contrasta los diferentes puntos de vista encontrados, presentando la información en un orden lógico (de general a particular) a partir de estudios seminales. Aunque existe un gran cuerpo teórico en las finanzas corporativas y en marketing acerca de las distintas métricas sobre el retorno de la inversión, para el tema puntual del social media ROI, no existen estudios concluyentes al respecto. De manera que, como no todos los estudios contribuyen al desarrollo de esta investigación, la revisión de literatura se limitó a aquellos trabajos que estudian únicamente la medición del retorno de la inversión en medios sociales.

La revisión sistemática de la literatura se realizó consultando principalmente dos bases de datos: Web of Science (WOS) y Scopus. El ejercicio se llevó a cabo siguiendo estos pasos: (1) búsqueda básica de los términos "social media" AND "Return on investment" ", limitada únicamente a la Colección principal de Web of Science. Estos términos fueron elegidos dado que son palabras claves en la mayoría de los artículos sobre el tema. Los resultados de la búsqueda fueron muy altos $\mathrm{y}$, aunque se utilizaron varios filtros, los trabajos encontrados no se refieren a la literatura en estudio. Lo anterior debido a que estos trabajos abordan el estudio del retorno de la inversión (ROI) desde una perspectiva muy general de las finanzas corporativas o en otros casos solo desde la perspectiva del marketing. 
Posteriormente la búsqueda se perfeccionó con los siguientes criterios: limitado al "idioma inglés", limitado al tipo de publicación "solo revistas" y limitado a la búsqueda "solo al título". Como resultado, se obtuvieron 64 registros. No obstante, y con el ánimo de precisar la revisión, la búsqueda se limitó solo a aquellas revistas que se consideran principales, tanto en ISI como en Scopus y, a su vez, contienen los artículos con el mayor número de citas. Además, se consultaron otros artículos, a los que se hizo referencia en los artículos revisados, y se incorporaron a la revisión de la literatura con el objetivo de fortalecer el cuerpo teórico que apoya la idea de investigación y permitir la identificación de la brecha en la literatura.

\section{Metodología}

Con el propósito de presentar los resultados de la revisión de la literatura de manera ordenada, estos fueron organizados bajo un enfoque Top- Down. En este sentido, en primer lugar, se analiza el retorno de la Inversión en Medios Sociales desde un contexto financiero amplio. Posteriormente, se realiza un análisis acerca de las mediciones comúnmente encontradas en la literatura.

Seguidamente, el análisis de la medición de ROI se separa en términos de sus componentes: esto es, por un lado, se presenta un análisis acerca de la medición de los costos y, por otro lado, se presentan los resultados en términos de la medición de los beneficios. De esta manera, los autores se aseguraron de abordar el concepto en su totalidad y poder presentar los resultados de manera detallada pero también desde una perspectiva integral.

\section{Discusión}

\section{Retorno de la Inversión en Medios Sociales (Social Media ROI)}

En un contexto amplio, es recurrente encontrar en la literatura la relación positiva entre los esfuerzos de las actividades de marketing y el rendimiento financiero (medido en términos de la Q de Tobin). Estudios han evidenciado que aquellas empresas que emplean una estrategia de marketing, tienen en promedio un rendimiento aproximado del 15\% mayor que el de aquellas empresas que no emplean una estrategia de marketing (Germann et al., 2015). Sin embargo, no existe mayor evidencia con el resultado que se obtiene de las inversiones hechas en medios sociales. Aunque estudios previos han demostrado que las inversiones en medios sociales ofrecen 
a las empresas nuevas formas de ganar valor comercial, también han identificado desafíos fundamentales relacionados con los mismos (Rokka et al., 2014).

Uno de los desafíos de los medios sociales es, precisamente, que puede ser difícil cuantificar el retorno de tal actividad, pues si bien muchas empresas usan los medios sociales como una herramienta que promueve el alcance de las fronteras, las empresas no miden si efectivamente su mensaje de marca, transmitido a través de las redes sociales, puede llegar a los consumidores finales o cuáles podrían ser los efectos finales del mensaje sobre la marca y el desempeño de los socios (Hamilton et al., 2016). Es por ello que, de la mano de la adopción generalizada de estas tecnologías sociales, los profesionales y los académicos han reconocido la necesidad de las organizaciones de cuantificar la contribución que generan las actividades de los medios sociales a los resultados financieros. Así mismo, la estimación del valor generado por los datos de las redes sociales para desarrollar su estrategia de marketing y administrar más eficientemente sus carteras de clientes (Nejada et al., 2016).

Al respecto, dos tipos diferentes de fórmulas se utilizan comúnmente para analizar la eficiencia de las actividades de marketing en medios sociales. El primero es un análisis de costo-beneficio que es utilizado para determinar si el costo de algo proporcionó un beneficio o una pérdida, es decir, su efectividad. La segunda, que se aproxima más a medir la eficiencia de modo cuantitativo, es el retorno de la inversión (ROI) (Crumpton, 2014). En este trabajo se pretende hacer una conceptualización de los elementos utilizados para calcular el ROI de las actividades de los medios sociales.

En términos generales, el Retorno de la Inversión (ROI) es una de las métricas de medición de rendimiento y evaluación más utilizadas en el análisis de negocios (Botchkarev y Andru, 2011). En el contexto del marketing resulta importante y necesario cuantificar la efectividad de los medios sociales más allá de observar solo las contribuciones en términos de interacciones y relaciones entre la firma y sus clientes (Michaelidou et al., 2011). De hecho, el efecto que tienen sobre las ventas las calificaciones o revisiones generadas por los usuarios en los sitios web, es un tema de estudio reciente, toda vez que esto impacta significativamente la forma en que los consumidores toman decisiones de compra. Estudios empíricos han establecido que en general, estas 
evaluaciones están positivamente correlacionadas con las ventas, pero su eficacia difiere dependiendo de la plataforma, el producto y la métrica (Babić et al., 2016). De igual modo, existe evidencia que explica como el liderazgo de opinión y el contagio social dentro de las redes sociales afectan la adopción de un nuevo producto (Iyengar et al., 2011).

A pesar de que las marcas gastan recursos significativos en las redes sociales para conectarse con sus clientes, hay una comprensión limitada de cómo los consumidores se involucran con las marcas en estos sitios y cómo influye en su proceso de compra (Saboo et al., 2016). Así mismo, se ha identificado que muchas empresas poseen políticas ambiguas, mensajes inconsistentes y carecen de una estrategia definida de medios sociales (Hamilton et al., 2016). Tradicionalmente el valor de un producto se ha evaluado de acuerdo con los ingresos directos que el producto genera. Sin embargo, los productos no existen aisladamente sino que influyen en las ventas del otro, influencia que es especialmente evidente en los entornos de comercio electrónico en los que los productos se presentan a menudo como una colección de páginas web vinculadas por hipervínculos de recomendación, creando una red de productos a gran escala (Oestreicher-Singer et al., 2013). De manera similar ocurre con el caso de las redes sociales en las que, cada vez, hay más entendimiento de que el valor de un cliente para una empresa proviene no sólo de sus compras, sino también de su influencia social, es decir, del voz a voz (Libai et al., 2013).

Este mayor involucramiento de las empresas en la gestión de relaciones con los clientes a través de los medios sociales ha dado lugar al término "CRM social", el cual ha venido ganando relevancia en los últimos años (Malthouse et al., 2013). A pesar de su importancia, hay escasa evidencia sobre cómo las actividades de marketing en medios sociales influyen en la creación de la marca y en el comportamiento que adopten los consumidores hacia una marca (Godey et al., 2016). Inclusive, algunas investigaciones han demostrado cómo los entornos de medios sociales amplifican la necesidad de distintas prácticas de gestión corporativa y cómo los retos y las soluciones que surgen a raíz de la presencia en medios de comunicación social, varían entre empresas de diferentes sectores (Rokka et al., 2014). Aun así, el uso de métricas tanto de marketing como financieras, estarán en función de la estrategia de la empresa, de qué tanta orientación tenga hacia la métrica, el tipo de actividad, así como de las características empresariales y ambientales (Mintz y Currim, 2013). 
En consecuencia, y debido a la importancia del tema, algunos estudios también han examinado cómo las empresas pueden aprovechar el poder de los medios de comunicación social y los esfuerzos de marketing en medios sociales, para obtener un mayor retorno de la inversión (Kumar et al., 2013). Lo anterior ha llevado a cuestionar cuál debe ser entonces la asignación presupuestaria óptima a través de cada canal de medios publicitarios, y ha conducido a evaluar cuál es la eficacia relativa de los medios de publicidad múltiples; es decir, cuáles medios influyen significativamente en los resultados de las compras y cuáles no (Danaher y Dagger, 2013). El dilema de la medición del retorno de las inversiones hechas en medios sociales ha suscitado mucho debate en los últimos tiempos. A medida que más y más empresas adoptan el uso de plataformas en medios sociales para mostrar sus productos o servicios online, el cálculo de esta inversión se convierte en una tarea compleja, pero si la empresa no lo hace, pondrá en peligro su capacidad para demostrar la plena recompensa de su uso (McCann y Barlow, 2015).

\section{¿Cómo se mide el ROI de los Medios Sociales?}

En términos generales, los indicadores financieros proporcionan una evaluación de la contribución económica de los medios sociales en términos de la rentabilidad de la inversión; estos indicadores son derivados de los indicadores tradicionalmente utilizados para evaluar el desempeño de las organizaciones. La métrica es la misma, pero el objeto de medición se ha desplazado pasando de medir a la organización, para medir ahora el desempeño financiero de las inversiones realizadas en medios sociales (Agostino y Sidorova, 2016). La literatura sobre marketing sugiere que las actividades de los medios sociales están interrelacionadas y se influyen mutuamente generando un efecto cascada (Hamilton et al., 2016). Por ejemplo, el número de seguidores debería influir en el número de comentarios de medios sociales y el muestreo de productos (Hollebeek et al., 2014). Del mismo modo, las marcas con una comunidad activa de medios de comunicación social pueden atraer a más seguidores y dar lugar a un mayor muestreo de productos.

Por las razones mencionadas anteriormente algunos autores han establecido que las actividades de las redes sociales se refuerzan entre sí, de manera que el aumento en una de las actividades hace

que la marca sea atractiva para los consumidores y, a su vez, influye positivamente en otras actividades de medios sociales (Kietzmann et al., 2011). Esta interrelación entre las distintas 
actividades de medios sociales, es justamente lo que dificulta la estimación precisa del retorno de las inversiones hechas en este tipo de actividades.

En general, el ROI es estimado de la siguiente manera: (Beneficios - Costos) / Costos. Esta medición pretende analizar, en términos porcentuales, la eficacia de la inversión para generar beneficios. Sin embargo, no es común en la literatura encontrar estudios que evalúen los aspectos financieros de las actividades llevadas a cabo en medios sociales, por lo que el trabajo desarrollado por Kumar et al. (2013) constituye una clara excepción. En su estudio, los autores proponen un nuevo marco para entender el flujo y la influencia del voz a voz y el beneficio financiero que el marketing de medios sociales puede proporcionar a una organización; su importancia radica en el hecho de que ignorar el voz a voz conduce a pérdidas significativas de ganancias para las empresas (Zubcsek y Sarvary, 2011).

Otra dificultad en la medición del ROI de marketing en medios sociales, es determinar si en efecto, estos medios resultaron en una venta o simplemente contribuyeron a ella. Lo anterior crea entonces un problema de endogeneidad: ¿El marketing de los medios sociales impulsó y contribuyó a la venta o simplemente influyó en el usuario final para comprar un producto? (Zubcsek y Sarvary, 2011). Aunque el efecto del contenido generado por la empresa en las redes sociales ha demostrado tener un efecto positivo y significativo sobre el gasto de los clientes y el comportamiento de compra cruzada (Kumar et al., 2016), persiste una generalizada falta de consenso sobre si el ROI es de hecho una métrica financiera o de marketing (Bendle y Bagga, 2016).

Por otra parte, y ante la dificultad que representa la medición del ROI, algunos autores han sugerido que en lugar de calcular el rendimiento de la inversión en medios sociales, los gerentes deben evaluar las motivaciones que tienen los consumidores para utilizar los medios de comunicación social y medir las inversiones de medios de comunicación social que hacen los clientes a medida que se involucran con las marcas de los vendedores, ya que la medición de las inversiones de los clientes en una relación de medios sociales revela la probabilidad de una recompensa a largo plazo, no sólo los resultados a corto plazo (Hoffman y Fodor, 2010). 
No obstante, la capacidad de demostrar el impacto de la acción de marketing en el desempeño financiero de la empresa es crucial para evaluar, justificar y optimizar el gasto de los recursos de marketing de una empresa (Dotson y Allenby, 2010). Reconociendo entonces las limitantes que la literatura plantea para el uso del ROI como medida de la eficiencia de las actividades de marketing, se presenta una conceptualización de sus componentes (costos y beneficios) con el fin de detallar los elementos necesarios para su cálculo y contribuir a la identificación cuidadosa de los mismos.

\section{Estimación de los Costos e Inversiones}

El retorno de la inversión (ROI) es una medida popular y potencialmente importante que permite comparar inversiones heterogéneas (Bendle y Bagga, 2016). Sin embargo, lo primero que se debe hacer antes de invertir recursos en una actividad que involucre medios sociales es establecer los objetivos, es decir, definir lo qué se espera lograr de una campaña en medios sociales (Hoffman y Fodor, 2010). Posteriormente, deben ser considerados también los recursos necesarios para implementar y utilizar las distintas plataformas y cómo podrá ser medido si la adopción de dichos medios permite alcanzar los objetivos de negocio que han sido planteados por la organización (McCann y Barlow, 2015).

Los costos generalmente se consideran la parte más fácil del cálculo del ROI (Botchkarev y Andru, 2011). En general, la estimación de las inversiones debe responder a una pregunta: ¿Cuáles son los costos asociados con los medios sociales? Por ello, para estimar este cálculo la mayoría inician midiendo el costo de lanzar un blog, por ejemplo, y luego buscan calcular el rendimiento de las ventas, es decir, el resultado de esa inversión (Hoffman y Fodor, 2010). De acuerdo con Botchkarev y Andru (2011), los costos asociados con un programa de medios sociales podrían ser enmarcados en las siguientes tres categorías: (a) costos de las tecnologías de la información, (b) costos de mano de obra y (c) costos de entrenamiento.

Los costos de las tecnologías de la información comprenden: el costo del software, las licencias (tanto la suscripción inicial como el mantenimiento anual), el costo del tiempo invertido para la creación de contenido de los materiales que se publicarán, costo del tiempo utilizado para la implementación o envío de materiales, costo del tiempo utilizado para responder a los usuarios e interactuar en el contexto del tema. En segundo lugar, se encuentran los costos de mano de obra 
que se refieren a los salarios y demás beneficios del recurso humano vinculado a la operación de los medios sociales; también deben ser incluidos en este rubro los gastos por servicios de consultoría. Finalmente, se encuentran los costos de entrenamiento que son aquellos que incluyen todos los gastos referentes a la capacitación del personal o a la formación del usuario final de un área de programación cuando es llevada a cabo por un tercero (Botchkarev y Andru, 2011).

Es de considerar que mover el presupuesto del marketing convencional a la publicidad más digital, social y móvil requiere la aceptación de todos los niveles de la jerarquía de la empresa. Algunas empresas buscan hacer pequeños cambios iniciales en su gasto en medios sociales, y luego determinar el retorno de estas inversiones (ROI). Sin embargo, el retorno de la inversión es difícil de demostrar, dejando a los altos directivos relativamente escépticos. Por lo tanto, los líderes de marketing tienen la responsabilidad de educar a su equipo y en lo posible, traer nuevos directores de organizaciones digitales para ayudar a estimular el pensamiento innovador y emprendedor (Grewal et al., 2016).

\section{Estimación de los Beneficios}

Antes de realizar una inversión en medios sociales, lo primero que debe responderse es la siguiente pregunta ¿Qué retorno se espera obtener?, esto es, definir en términos del negocio y de la misión, cuáles serán las metas, los objetivos y las métricas que serán utilizadas, por la organización antes de su entrada en el ámbito de los medios sociales (Hoffman y Fodor, 2010). Aunque sólo las ganancias financieras pueden medirse en valor monetario, las ganancias no financieras de los medios de comunicación social pueden ser igualmente beneficiosas. Sin embargo, aunque esto puede ser ventajoso para cualquier negocio, la medición de estos beneficios intangibles es compleja. Si bien las métricas tales como un aumento en los amigos de Facebook o un aumento en los seguidores de Twitter no necesariamente se relacionan con el incremento en las ventas, la presencia de una empresa a través de las plataformas de medios sociales puede llevar a ganancias financieras (McCann y Barlow, 2015).

De acuerdo con Botchkarev y Andru (2011), los beneficios asociados con un programa de medios sociales podrían ser enmarcados en las siguientes categorías: (a) ahorro o eliminación de costos, 
(b) incremento en ventas, (c) mejora de los ingresos y (d) protección de los ingresos. Sin embargo, un requisito crítico para calcular el ROI es conocer el beneficio neto generado por una decisión de inversión específica, pues no es suficiente con conocer los beneficios totales y la inversión. Para calcular el ROI con precisión, se hace necesario estimar la fracción de los beneficios atribuibles a la inversión, es decir, cuál sería el beneficio si no se hubiera realizado la inversión, lo que se conoce como beneficio incremental (Bendle y Bagga, 2016).

Si bien hay evidencia inicial acerca de las contribuciones financieras de los programas de medios sociales (Kumar et al., 2010), sigue siendo necesario mejorar el entendimiento de cómo generar valor monetario de estos programas; los responsables de la toma de decisiones de marketing son cada vez más conscientes de la importancia de la maximización del valor para los accionistas, lo que exige una evaluación de los efectos a largo plazo de sus acciones sobre la respuesta del mercado de productos y la respuesta de los inversores (Joshi y Hanssens, 2010). Finalmente, y dado que ninguna métrica por sí sola capta suficientemente los fenómenos importantes y diversos en los medios sociales, los gerentes necesitan un enfoque sistemático para identificar y construir las métricas apropiadas, relacionando la métrica de marketing con los resultados financieros, lo cual es un requisito previo para la eficiente gestión de los medios sociales (Peters et al., 2013).

Por ejemplo, para medir el impacto del voz a voz en las redes sociales, la literatura sugiere que lo primero que se debe hacer es identificar quienes son los influenciadores, para lo cual es necesario entender cómo la información fluye a través de la red, es decir, analizar los datos de ventas que describen el comportamiento de compra de cada individuo, lo cual es posible lograr encontrando coincidencias entre el tipo de información y la influencia que cada individuo tiene en los demás (Kumar et al., 2013). Sin embargo, la falta de datos con los que hacer proyecciones sobre el comportamiento de compra futuro de los clientes obtenidos de las redes sociales, es otro desafío. La organización puede albergar gigabytes de datos, pero eso no significa que los datos sean particularmente útiles para el análisis financiero de las comunicaciones de redes sociales (Cronin, 2014). 


\section{Conclusiones}

Los medios sociales son más que otra forma de medios, son un movimiento en el que los consumidores y las empresas participan en un diálogo no estructurado, descubrimiento y entrega de información, y toman decisiones de compra, donde la emoción juega un papel fundamental y moldea la información que se transmite (Berger y Milkman, 2012). Son el comercio donde la gente compra, pero también es la comunidad donde la gente se reúne, se conecta y se comunica. Es un matrimonio complejo de psicología y tecnología, cuyo impacto en el valor de una organización no puede ser subestimado (Berger y Schwartz, 2011). La elección de cuándo participar, cómo manejarlo y cómo medirlo es compleja pero no imposible. Estos cuestionamientos parecen no tener una única respuesta porque el contexto y la dinámica del mercado son variables importantes en este tipo de decisiones (Nair, 2011).

Por tanto, la medición de los medios tradicionales parece casi rara en el entorno de marketing de medios sociales cada vez más complejo y dinámico de hoy (Hoffman y Fodor, 2010). Por ello, se puede concluir que en ausencia del marketing tradicional y de las mejores prácticas estandarizadas, algunos recurren al retorno de la inversión (ROI) como medio para probar las contribuciones que los medios sociales generan. No obstante, muchos beneficios proporcionados por las redes sociales no se miden fácilmente en dólares y centavos, por lo que el retorno de la inversión en marketing de medios de comunicación social debe vincular directamente la meta de su presencia en los medios sociales y los objetivos de la organización (Harrigan y Hulbert, 2011).

Por otro lado, la literatura evidencia que entre los gerentes de marketing parece no existir consenso respecto a lo que es el ROI. Una campaña para crear conciencia puede tener un ROI positivo, por ejemplo, pero los responsables de marketing no pueden demostrar esto simplemente señalando niveles más altos de conciencia. Para calcular el ROI, debe haber un retorno (un beneficio asociado con la inversión) y una inversión, pues a menos que tenga ambos, no se podría calcular ROI (Bendle y Bagga, 2016). Así las cosas, el cálculo del ROI sigue representando, sin duda, un gran desafío para los académicos y profesionales de marketing. 


\section{Otras consideraciones}

El concepto de medios sociales está en la parte superior de la agenda para muchos ejecutivos de negocios de hoy. Los tomadores de decisiones, así como los consultores, tratan de identificar maneras en que las empresas pueden hacer uso rentable de aplicaciones como Wikipedia, YouTube, Facebook, Second Life y Twitter (Kaplan y Haenlein, 2010). No obstante, se argumenta que cuando la tecnología está impulsando la comercialización en la práctica, se presta significativamente menos atención en teoría y educación. Por lo tanto, los graduados de marketing que se producen de las universidades suelen carecer de las habilidades que los responsables de marketing del siglo XXI requieren (Harrigan y Hulbert, 2011).

Sin embargo, y a pesar de este interés, parece haber una comprensión muy limitada de lo que el término medios sociales significa exactamente. Es decir, existe una aparente confusión entre los gerentes y los investigadores académicos por aquello que puntualmente debe incluirse bajo el término de medios sociales, y cómo los medios sociales difieren de los aparentemente intercambiables conceptos relacionados de la Web 2.0 y contenido generado por el usuario (CGU). Por lo tanto, tendría sentido ahondar en estudios que proporcionen evidencia empírica con respecto a los medios sociales y lo que éstos verdaderamente significan (Kaplan y Haenlein, 2010). Al parecer existe una desconexión entre la educación en marketing y lo que se hace en la práctica de marketing, por lo que se espera que los académicos del tema deban enseñar más en línea con lo que realmente se está practicando por los profesionales de marketing en el siglo XXI (Harrigan y Hulbert, 2011).

\section{Referencias}

(1) Agostino, D., \& Sidorova, Y. (2016). A performance measurement system to quantify the contribution of social media: new requirements for metrics and methods. Measuring Business Excellence, 20(2), 38-51. https://doi.org/10.1108/MBE-05-2015-0030

(2) Babić, A., Sotgiu, F., De Valck, K., \& Bijmolt, T. H. (2016). The effect of electronic word of mouth on sales: a meta-analytic review of platform, product, and metric factors. Journal of Marketing Research, 53(3), 297-318. https://doi.org/10.1509/jmr.14.0380 
(3) Bendle, N., \& Bagga, C. (2016). The metrics that marketers muddle. MIT Sloan Management Review, 57(3), 72-82.

(4) Berger, J., \& Milkman, K. L. (2012). What makes online content viral? Journal of Marketing Research, 49(2), 192-205. https://doi.org/10.1509/jmr.10.0353

(5) Berger, J., \& Schwartz, E. M. (2011). What drives immediate and ongoing word of mouth? Journal of Marketing Research, 48(5), 869-880. https://doi.org/10.1509/jmkr.48.5.869

(6) Botchkarev, A., \& Andru, P. (2011). A return on investment as a metric for evaluating information systems: taxonomy and application. Interdisciplinary Journal of Information, Knowledge, and Management, 6, 245-269. https://doi.org/10.28945/1535

(7) Cronin, J. J. (2014). Teaching ROI analysis in an era of social media. Journal of Advertising Education, 18(2), 28-35. https://doi.org/10.1177/109804821401800205

(8) Crumpton, M. A. (2014). Accounting for the cost of social media. The Bottom Line: Managing library finances, 27(3), 96-100.

(9) Danaher, P., \& Dagger, T. S. (2013). Comparing the relative effectiveness of advertising channels: a case study of a multimedia blitz campaign. Journal of Marketing Research, 50(4), 517-534. https://doi.org/10.1509/jmr.12.0241

(10)Dotson, J., \& Allenby, G. M. (2010). Investigating the strategic influence of customer and employee satisfaction on firm financial performance. Marketing Science, 29(5), 895-908. http://www.jstor.org/stable/40864672

(11) Germann, F., Ebbes, P., \& Grewal, R. (2015). The chief marketing officer matters! Journal of Marketing, 79(3), 1-22. https://doi.org/10.1509/jm.14.0244

(12) Godey, B., Manthiou, A., Pederzoli, D., Rokka, J., Aiello, G., Donvito, R., \& Singh, R. (2016). Social media marketing efforts of luxury brands: Influence on brand equity and consumer behavior. Journal of business research,69(12), 5833-5841. https://doi.org/10.1016/j.jbusres.2016.04.181

(13) Grewal, D., Bart, Y., Spann, M., \& Zubcsek, P. (2016). Mobile advertising: a framework and research agenda. Journal of Interactive Marketing, 34, 3-14. https://doi.org/10.1016/j.intmar.2016.03.003

(14) Hamilton, M., Kaltcheva, V. D., \& Rohm, A. J. (2016). Social media and value creation: the role of interaction satisfaction and interaction immersion. Journal of Interactive Marketing, 36, 121-133. https://doi.org/10.1016/j.intmar.2016.07.001 
(15)Hanna, R., Rohm, A., \& Crittenden, V. (2011). We're all connected: the power of the social media ecosystem. Business Horizons, 54, 265-273.

(16) Harrigan, P., \& Hulbert, B. (2011). How can marketing academics serve marketing practice? The new marketing DNA as a model for marketing education. Journal of Marketing Education, 33(3), 253-272. https://doi.org/10.1177/0273475311420234

(17) Hoffman, D., \& Fodor, M. (2010). Can you measure the ROI of your social media marketing? MIT Sloan Management Review, 52(1), 40-49.

(18) Hollebeek, L. D., Glynn, M. S., \& Brodie, R. J. (2014). Consumer brand engagement in social media: conceptualization, scale development and validation. Journal of Interactive Marketing, 28(2), 149-165. https://doi.org/10.1016/j.intmar.2013.12.002

(19) Iyengar, R., Van den Bulte, C., \& Valente, T. W. (2011). Opinion leadership and social contagion in new product diffusion. Marketing Science, 30(2), 195-212. https://doi.org/10.1287/mksc.1100.0566

(20) Joshi, A., \& Hanssens, D. M. (2010). The direct and indirect effects of advertising spending on firm value. Journal of Marketing, 74(1), 20-33. https://doi.org/10.1509/jmkg.74.1.20

(21)Kaplan, A., \& Haenlein, M. (2010). Users of the world, unite! The challenges and opportunities of Social Media. Business Horizons, 53, 59-68. https://doi.org/10.1016/j.bushor.2009.09.003

(22) Kietzmann, J. H., Hermkens, K., McCarthy, I. P., \& Silvestre, B. S. (2011). Social media? Get serious! Understanding the functional building blocks of social media. Business Horizons, 54(3), 241-251. https://doi.org/10.1016/j.bushor.2011.01.005

(23) Kumar, A., Bezawada, R., Rishika, R., Janakiraman, R., \& Kannan, P. K. (2016). From social to sale: the effects of firm-generated content in social media on customer behavior. Journal of Marketing, 80(1), 7-22. https://doi.org/10.1509/jm.14.0249

(24) Kumar, V., Bhaskaran, V., Mirchandani, R., \& Shah, M. (2013). Creating a measurable social media marketing strategy: increasing the value and ROI of intangibles and tangibles for Hokey Pokey. Marketing Science, 32(2), 194-212. https://doi.org/10.1287/mksc.1120.0768

(25) Kumar, V., Petersen, A., \& Leone, R. (2010). Driving profitability by encouraging customer referrals: Who, when, and how. Journal of Marketing, 74, 1-17. https://doi.org/10.1509/jmkg.74.5.001 
(26) Kumar, V., Zhang, X. (Alan), \& Luo, A. (2014). Modeling customer opt-in and opt-out in a permission-based marketing context. Journal of Marketing Research, 51(4), 403-419. https://doi.org/10.1509/jmr.13.0169

(27)Labrecque, L. I. (2014). Fostering consumer-brand relationships in social media environments:the role of parasocial interaction. Journal of Interactive Marketing, 28, 134148. https://doi.org/10.1016/j.intmar.2013.12.003

(28) Lamberton, C., \& Stephen, A. T. (2016). A Thematic Exploration of Digital, Social Media, and Mobile Marketing: Research Evolution from 2000 to 2015 and an Agenda for Future Inquiry. Journal of Marketing, 80, 146-172. https://doi.org/10.1509/jm.15.0415

(29)Libai, B., Muller, E., \& Peres, R. (2013). Decomposing the value of word-of-mouth seeding programs: acceleration vs. expansion. Journal of Marketing Research, 50(2), 161-176. https://doi.org/10.1509/jmr.11.0305

(30) Malthouse, E., Haenlein, M., Skiera, B., Wege, E., \& Zhang, M. (2013). Managing customer relationships in the social media era: introducing the social CRM house. Journal of Interactive Marketing, 27(4), 270-280. https://doi.org/10.1016/j.intmar.2013.09.008

(31) McCann, M., \& Barlow, A. (2015). Use and measurement of social media for SMEs. Journal of Small Business and Enterprise Development, 22(2), $273 \quad$ - 287. https://doi.org/10.1108/JSBED-08-2012-0096

(32) Michaelidou, N., Siamagka, N., \& Christodoulides, G. (2011). Usage, barriers and measurement of social media marketing: an exploratory investigation of small and medium B2B brands. Industrial Marketing Management, 40(7), 1153-1159. https://doi.org/10.1016/j.indmarman.2011.09.009

(33) Mintz, O., \& Currim, I. S. (2013). What drives managerial use of marketing and financial metrics and does metric use impact marketing mix performance. Journal of Marketing, 77(2), 17-40.

(34) Nair, M. (2011). Understanding and measuring the value of social media. The Journal of Corporate Accounting and Finance, 22(3), 45-51. https://doi.org/10.1002/jcaf.20674

(35) Nejada, M. G., Aminib, M., \& Sherrel, D. L. (2016). The profit impact of revenue heterogeneity and assortativity in the presence of negative word-of-mouth . International Journal of Research in Marketing, 33(3), 656-673. https://doi.org/10.1016/j.ijresmar.2015.11.005 
(36) Oestreicher-Singer, G., Libai, B., Sivan, L., Carmi, E., \& Yassin, O. (2013). The network value of products. Journal of Marketing, 77(3), 1-14. https://doi.org/10.1509/jm.11.0400

(37)Peters, K., Chen, Y., Kaplan, A., Ognibeni, B., \& Pauwels, K. (2013). Social Media Metrics - A Framework and Guidelines for Managing Social Media. Journal of Interactive Marketing, 27(4), 281-298. https://doi.org/10.1016/j.intmar.2013.09.007

(38)Rapp, A., Beitelspacher, L., Grewal, D., \& Hughes, D. (2013). Understanding social media effects across seller, retailer, and consumer interactions. Journal of the Academy of Marketing Science, 41(5), 547-566. https://doi.org/10.1007/s11747-013-0326-9

(39) Rokka, J., Karlsson, K., \& Tienari, J. (2014). Balancing acts: Managing employees and reputation in social media. Journal of Marketing Management, 30(7-8), 802-827. https://doi.org/10.1080/0267257X.2013.813577

(40) Saboo, A., Kumar, V., \& Ramani, G. (2016). Evaluating the impact of social media activities on human. International Journal of Research in Marketing, 33, 524-541. https://doi.org/10.1016/j.ijresmar.2015.02.007

(41) Weinberg, B. D., \& Pehlivan, E. (2011). Social spending: Managing the social media mix. Business Horizons, 54(3), 275-282. https://doi.org/10.1016/j.bushor.2011.01.008

(42)Zubcsek, P., \& Sarvary, M. (2011). Advertising to a social network. Quantitative Marketing and Economics, 9(1), 71-107. https://doi.org/10.1007/s11129-010-9093-9

Cómo citar este artículo: Vergara, J., Álvarez, P. y Serna, M. (2021). El retorno de la inversión en medios sociales (Social Media ROI). Una revisión de literatura. Tendencias, 22(2), 331-348. https://doi.org/10.22267/rtend.212202.179 